\title{
Efficacy of tocilizumab treatment in severely ill COVID-19 patients
}

Jie Zhao, Wei Cui and Bao-ping Tian ${ }^{*}$

Keywords: Tocilizumab, COVID-19, Efficacy, Meta-analysis

The current coronavirus disease 2019 (COVID-19) pandemic induced by severe acute respiratory syndrome coronavirus 2 (SARS-CoV-2) has already caused a global increase in hospitalizations and deaths. Unfortunately, effective medicines to fight this disease, especially in the severely ill patients, are still lacking [1]. Tocilizumab, a humanized monoclonal antibody used in rheumatoid arthritis treatment, might also be effective in treating severe COVID-19 as it could selectively target the interleukin-6 (IL-6) receptor [2]. Considering the uncertain efficacy of tocilizumab treatment in severe COVID-19, we conducted a systematic review and meta-analysis to clarify this added effect of tocilizumab.

We performed a systematic search of PubMed, Embase, Medline, Cochrane, and CNKI database through 25 July 2020, using the following search terms alone or in combination: (1) "COVID-19," (2) "coronavirus," (3) "SARS-CoV-2," (4) "COVID," (5) "antiinterleukin-6 receptor antibodies," (6) "anti-IL-6 receptor antibodies," (7) "anti-IL-6," (8) "tocilizumab," (9)"sarilumab," and (10) "siltuximab." Clinical trials regarding tocilizumab as a therapeutic intervention were selected. Two independent investigators selected eligible trials and extracted data from articles. Discrepancies in screening/data extraction were addressed by group discussion. Proportional variables were measured by odds ratio (OR) and corresponding 95\% confidence intervals $(\mathrm{CI}) . P$ values $<0.05$ were

\footnotetext{
*Correspondence: TianBP@zju.edu.cn

Department of Critical Care Medicine, The Second Affiliated Hospital, Zhejiang University School of Medicine, 88 Jiefang Rd., Hangzhou 310009, Zhejiang, China
}

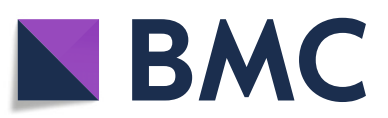

(c) The Author(s). 2020 Open Access This article is licensed under a Creative Commons Attribution 4.0 International License, which permits use, sharing, adaptation, distribution and reproduction in any medium or format, as long as you give appropriate credit to the original author(s) and the source, provide a link to the Creative Commons licence, and indicate if changes were made. The images or other third party material in this article are included in the article's Creative Commons licence, unless indicated otherwise in a credit line to the material. If material is not included in the article's Creative Commons licence and your intended use is not permitted by statutory regulation or exceeds the permitted use, you will need to obtain permission directly from the copyright holder. To view a copy of this licence, visit http://creativecommons.org/licenses/by/4.0/. The Creative Commons Public Domain Dedication waiver (http://creativecommons.org/publicdomain/zero/1.0/) applies to the data made available in this article, unless otherwise stated in a credit line to the data. considered statistically significant. Significant heterogeneity $\left(P<0.10\right.$ or $\left.I^{2} \geq 50 \%\right)$ was evaluated by chisquare and $I^{2}$ tests in a fixed-effect model. The comparison of the outcome between tocilizumab and control was conducted by using Review Manager 5.4 (Revman, The Cochrane Collaboration, Oxford, UK).

Finally, 10 studies involving 1675 severe COVID-19 patients were included, among which only one trial was a randomized controlled trial, while the rest were all retrospective cohort studies. These studies included COVID-19 patients who were older/elderly (mean/median age $\geq 52$ years) in America, Europe, and India, among whom 675 patients received tocilizumab, while 1000 patients underwent standard care. Severe COVID-19 patients received tocilizumab via intravenous or subcutaneous formulation, while doses and administration time points varied. Standard care included hydroxychloroquine, lopinavir/ritonavir, remdesivir, azithromycin, low weight heparin, and/or methylprednisolone, among others (Table 1). Our metaanalysis result revealed a significant difference in mortality between tocilizumab group $(132 / 675,19.5 \%)$ and control group $(283 / 1000,28.3 \%)$ in the fixed-effect model (OR, $0.47 ; 95 \% \mathrm{Cl}, 0.36-0.60 ; P<0.00001)$, suggesting efficacy of tocilizumab treatment for severe COVID-19. However, high heterogeneity was also observed $\left(I^{2}=74 \%, P<\right.$ $0.0001)$ as shown in Fig. 1. SARS-CoV-2 infection might cause a hyperimmune response associated with acute respiratory distress (ARDS), the latteris a leading cause of death for severe COVID-19 [3]. Uncontrolled immune activation would result in cytokine storm, also known as cytokine release syndrome (CRS), appearing as overproduction of pro-inflammatory cytokines and chemokines [4]. Severe COVID-19 patients always present elevated 
Table 1 Study characteristics and demographics of included severely ill coronavirus disease 2019 (COVID-19) patients

\begin{tabular}{|c|c|c|c|c|c|c|c|c|}
\hline Article & Study design & Country & $\begin{array}{l}\text { Total } \\
\text { patients }\end{array}$ & $\begin{array}{l}\text { Mean/ } \\
\text { median age } \\
\text { (years) }\end{array}$ & Standard care & $\begin{array}{l}\text { Tocilizumab } \\
\text { treatment }\end{array}$ & Patients category & $\begin{array}{l}\text { Primary } \\
\text { outcomes }\end{array}$ \\
\hline $\begin{array}{l}\text { Campochiaro C } \\
\text { Eur J Intern Med } \\
2020\end{array}$ & $\begin{array}{l}\text { Single-center } \\
\text { retrospective } \\
\text { cohort study }\end{array}$ & Italy & 65 & $\begin{array}{l}60 \text { (control) } \\
64 \\
\text { (tocilizumab) }\end{array}$ & $\begin{array}{l}\text { Hydroxychloroquine, } \\
\text { lopinavir/ritonavir, } \\
\text { ceftriaxone, } \\
\text { azithromycin }\end{array}$ & $\begin{array}{l}\text { First intravenous } \\
400 \mathrm{mg} \text {, second } \\
400 \mathrm{mg} \text { was } \\
\text { administered due } \\
\text { to progressive } \\
\text { respiratory } \\
\text { worsening }\end{array}$ & $\begin{array}{l}\text { Severe COVID-19 } \\
\text { patients with } \\
\text { hyper-inflammatory } \\
\text { features admitted } \\
\text { outside ICU requir- } \\
\text { ing NIV and/or } \\
\text { high-flow supple- } \\
\text { mental } \mathrm{O}_{2}\end{array}$ & $\begin{array}{l}\text { Safety, } \\
\text { efficacy }\end{array}$ \\
\hline $\begin{array}{l}\text { Capra R } \\
\text { Eur J Intern Med } \\
2020\end{array}$ & $\begin{array}{l}\text { Retrospective } \\
\text { observational } \\
\text { study }\end{array}$ & Italy & 85 & $\begin{array}{l}70 \text { (control) } \\
63 \\
\text { (tocilizumab) }\end{array}$ & $\begin{array}{l}\text { Hydroxychloroquine, } \\
\text { lopinavir/ritonavir }\end{array}$ & $\begin{array}{l}\text { Tocilizumab once } \\
\text { within } 4 \text { days }\end{array}$ & $\begin{array}{l}\text { COVID-19-related } \\
\text { pneumonia and } \\
\text { respiratory failure, } \\
\text { not needing } \\
\text { mechanical } \\
\text { ventilation }\end{array}$ & Survival rate \\
\hline $\begin{array}{l}\text { Colaneri M } \\
\text { Microorganisms } \\
2020\end{array}$ & $\begin{array}{l}\text { Retrospective } \\
\text { case-control } \\
\text { study }\end{array}$ & Italy & 112 & $\begin{array}{l}64 \text { (control) } \\
62 \\
\text { (tocilizumab) }\end{array}$ & $\begin{array}{l}\text { Hydroxychloroquine, } \\
\text { azithromycin, low } \\
\text { weight heparin, } \\
\text { methylprednisolone }\end{array}$ & $\begin{array}{l}\text { First administration } \\
\text { was } 8 \mathrm{mg} / \mathrm{kg} \text { (up to } \\
\text { a maximum } 800 \\
\text { mg per dose) } \\
\text { intravenously, } \\
\text { repeated after } 12 \mathrm{~h}\end{array}$ & $\begin{array}{l}\text { Critically ill patients } \\
\text { with severe COVID- } \\
19 \text { pneumonia }\end{array}$ & $\begin{array}{l}\text { Admission to } \\
\text { the ICU and } \\
\text { 7-day mortal- } \\
\text { ity rate }\end{array}$ \\
\hline $\begin{array}{l}\text { Gokhale Y } \\
\text { EClinicalMedicine } \\
2020\end{array}$ & $\begin{array}{l}\text { Retrospective } \\
\text { cohort study }\end{array}$ & India & 161 & $\begin{array}{l}55 \text { (control) } \\
52 \\
\text { (tocilizumab) }\end{array}$ & $\begin{array}{l}\text { Antibiotics, } \\
\text { hydroxychloroquine } \\
\text { oseltamivir, low } \\
\text { molecular weight } \\
\text { heparin, } \\
\text { methylprednisolone }\end{array}$ & $\begin{array}{l}\text { A single } \\
\text { intravenous dose of } \\
400 \mathrm{mg}\end{array}$ & $\begin{array}{l}\text { COVID-19 with } \\
\text { oxygen saturation } \\
\text { of } 94 \% \text { or less } \\
\text { despite giving } \\
\text { supplemental } \\
\text { oxygen of } 15 \mathrm{~L} / \mathrm{min} \\
\text { via non-rebreathing } \\
\text { mask or } \mathrm{PaO} 2 / \mathrm{FiO} 2 \\
\text { ratio of less than } \\
200\end{array}$ & Death \\
\hline $\begin{array}{l}\text { Guaraldi G } \\
\text { Lancet } \\
\text { Rheumatol } \\
2020\end{array}$ & $\begin{array}{l}\text { Retrospective } \\
\text { observational } \\
\text { cohort study }\end{array}$ & Italy & 544 & $\begin{array}{l}69 \text { (control) } \\
64 \\
\text { (tocilizumab) }\end{array}$ & $\begin{array}{l}\text { Oxygen supply to } \\
\text { target } \mathrm{SaO}_{2} \text { reaching } \\
\text { at least } 90 \% \text {, } \\
\text { hydroxychloroquine, } \\
\text { azithromycin at the } \\
\text { physician's discretion } \\
\text { when suspecting a } \\
\text { bacterial respiratory } \\
\text { super-infection, lopi- } \\
\text { navir-ritonavir or } \\
\text { darunavir-cobicistat, } \\
\text { low molecular } \\
\text { weight heparin }\end{array}$ & $\begin{array}{l}\text { Intravenous } \\
\text { tocilizumab was } \\
\text { administered at } 8 \\
\mathrm{mg} / \mathrm{kg} \text { bodyweight } \\
\text { (up to a maximum } \\
\text { of } 800 \mathrm{mg} \text { ) } \\
\text { administered twice, } \\
12 \mathrm{~h} \text { apart; the } \\
\text { subcutaneous } \\
\text { formulation was } \\
\text { used when there } \\
\text { was a shortage of } \\
\text { the intravenous } \\
\text { formulation, at a } \\
\text { dose of } 162 \mathrm{mg} \\
\text { administered in } \\
\text { two simultaneous } \\
\text { doses, one in each } \\
\text { thigh }\end{array}$ & $\begin{array}{l}\text { Severe pneumonia } \\
\text { defined at least one } \\
\text { of the following: } \\
\text { presence of a } \\
\text { respiratory rate of } \\
30 \text { or more breaths } \\
\text { per minute, } \\
\text { peripheral blood } \\
\mathrm{SaO}_{2} \text { of less than } \\
93 \% \text { in room air, a } \\
\text { ratio of } \mathrm{PaO}_{2} \text { to } \\
\mathrm{FiO}_{2} \text { of less than } \\
300 \text { mmHg in room } \\
\text { air, and lung } \\
\text { infiltrates of more } \\
\text { than } 50 \% \text { within } \\
24-48 \mathrm{~h} \text {, according } \\
\text { to Chinese } \\
\text { management } \\
\text { guidelines for } \\
\text { COVID-19 }\end{array}$ & $\begin{array}{l}\text { Death or } \\
\text { invasive } \\
\text { mechanical } \\
\text { ventilation }\end{array}$ \\
\hline $\begin{array}{l}\text { Klopfenstein T } \\
\text { Med Mal Infect } \\
2020\end{array}$ & $\begin{array}{l}\text { Retrospective } \\
\text { case-control } \\
\text { study }\end{array}$ & France & 45 & $\begin{array}{l}71 \text { (control) } \\
77 \\
\text { (tocilizumab) }\end{array}$ & $\begin{array}{l}\text { Hydroxychloroquine } \\
\text { or lopinavir-ritonavir, } \\
\text { antibiotics, less com- } \\
\text { monly } \\
\text { corticosteroids }\end{array}$ & $\begin{array}{l}1 \text { or } 2 \text { doses (no } \\
\text { detail was } \\
\text { reported) }\end{array}$ & $\begin{array}{l}\text { All critically COVID- } \\
19 \text { patients in toci- } \\
\text { lizumab group, } \\
\text { fewer critically ill } \\
\text { patients in control }\end{array}$ & $\begin{array}{l}\text { Death and/or } \\
\mathrm{ICU} \\
\text { admissions }\end{array}$ \\
\hline $\begin{array}{l}\text { Moreno-Pérez O } \\
\text { J Autoimmun } \\
2020\end{array}$ & $\begin{array}{l}\text { Retrospective } \\
\text { cohort study }\end{array}$ & Spain & 236 & $\begin{array}{l}57 \text { (control) } \\
62 \\
\text { (tocilizumab) }\end{array}$ & $\begin{array}{l}\text { No detail was } \\
\text { reported }\end{array}$ & $\begin{array}{l}\text { Initial } 600 \mathrm{mg} \text {, with } \\
\text { a second or third } \\
\text { dose }(400 \mathrm{mg}) \text { in } \\
\text { case of persistent } \\
\text { or progressive } \\
\text { disease }\end{array}$ & $\begin{array}{l}\text { Severe COVID-19 } \\
\text { pneumonia }\end{array}$ & $\begin{array}{l}\text { All-cause } \\
\text { mortality }\end{array}$ \\
\hline
\end{tabular}


Table 1 Study characteristics and demographics of included severely ill coronavirus disease 2019 (COVID-19) patients (Continued)

\begin{tabular}{|c|c|c|c|c|c|c|c|c|}
\hline Article & Study design & Country & $\begin{array}{l}\text { Total } \\
\text { patients }\end{array}$ & $\begin{array}{l}\text { Mean/ } \\
\text { median age } \\
\text { (years) }\end{array}$ & Standard care & $\begin{array}{l}\text { Tocilizumab } \\
\text { treatment }\end{array}$ & Patients category & $\begin{array}{l}\text { Primary } \\
\text { outcomes }\end{array}$ \\
\hline $\begin{array}{l}\text { Potere N } \\
\text { Ann Rheum Dis } \\
2020\end{array}$ & $\begin{array}{l}\text { Retrospective } \\
\text { case-control } \\
\text { study }\end{array}$ & Italy & 80 & $\begin{array}{l}54 \text { (control) } \\
56 \\
\text { (tocilizumab) }\end{array}$ & $\begin{array}{l}\text { Hydroxychloroquine, } \\
\text { darunavir/cobicistat, } \\
\text { lopinavir/ritonavir, } \\
\text { systemic } \\
\text { corticosteroid }\end{array}$ & $\begin{array}{l}324 \text { mg given as } \\
\text { two concomitant } \\
\text { subcutaneous } \\
\text { injections }\end{array}$ & $\begin{array}{l}\text { Severe COVID-19 } \\
\text { pneumonia with } \\
\text { hypoxemia (oxygen } \\
\text { saturation < } 90 \% \text { on } \\
\text { room air) requiring } \\
\text { supplemental oxy- } \\
\text { gen through nasal } \\
\text { cannulas or mask }\end{array}$ & $\begin{array}{l}\text { Requirement } \\
\text { of IMV or } \\
\text { death }\end{array}$ \\
\hline $\begin{array}{l}\text { Rojas-Marte GR } \\
\text { QJM: An } \\
\text { International } \\
\text { Journal of } \\
\text { Medicine } 2020\end{array}$ & $\begin{array}{l}\text { Retrospective, } \\
\text { case-control, } \\
\text { single-center } \\
\text { study }\end{array}$ & USA & 193 & $\begin{array}{l}62 \text { (control) } \\
59 \\
\text { (tocilizumab) }\end{array}$ & $\begin{array}{l}\text { Hydroxychloroquine, } \\
\text { azithromycin, } \\
\text { corticosteroids } \\
\text { anticoagulation, } \\
\text { remdesivir, } \\
\text { antibiotics for } \\
\text { suspected bacterial } \\
\text { infections, } \\
\text { vasopressors }\end{array}$ & $\begin{array}{l}\text { No detail was } \\
\text { reported }\end{array}$ & $\begin{array}{l}\text { Adult patients } \\
\text { hospitalized with } \\
\text { severe COVID-19 }\end{array}$ & $\begin{array}{l}\text { Overall } \\
\text { mortality rate }\end{array}$ \\
\hline $\begin{array}{l}\text { Somers EC } \\
\text { Clin Infect Dis } \\
2020\end{array}$ & $\begin{array}{l}\text { Randomized } \\
\text { controlled trial }\end{array}$ & USA & 154 & $\begin{array}{l}60 \text { (control) } \\
55 \\
\text { (tocilizumab) }\end{array}$ & $\begin{array}{l}\text { Hydroxychloroquine, } \\
\text { remdesivir, NSAIDs, } \\
\text { ACEI/ARB, } \\
\text { vasopressors, } \\
\text { anticoagulation } \\
\text { corticosteroid }\end{array}$ & $\begin{array}{l}\text { The standard } \\
\text { tocilizumab dose } \\
\text { was } 8 \mathrm{mg} / \mathrm{kg} \\
\text { (maximum } 800 \mathrm{mg} \text { ) } \\
\times 1 \text {, additional } \\
\text { doses were } \\
\text { discouraged }\end{array}$ & $\begin{array}{l}\text { Severe COVID-19 } \\
\text { patients requiring } \\
\text { mechanical } \\
\text { ventilation }\end{array}$ & $\begin{array}{l}\text { Survival } \\
\text { probability } \\
\text { after } \\
\text { intubation }\end{array}$ \\
\hline
\end{tabular}

inflammatory markers, among which the elevation of IL-6 is associated with severity of COVID-19 [5]. Besides, the upregulated expression of IL- 6 receptor (IL-6R) was also detected in COVID-19 patients [6]. Therefore, IL-6/IL6R might serve as a messenger not only for transmitting inflammatory signals throughout the lung and other organs but also by activating cellular signal pathway, thus causing ARDS and multiple organ dysfunction. It is reasonable to speculate that IL- 6 blockade is beneficial for avoiding poor prognosis.
Our meta-analysis had several limitations: (1) most included studies were retrospective analysis of cases, resulting in poor quality of the included studies; (2) the uniformity of the diagnostic criteria for severe COVID19 needs to be improved, and the extraction of related factors is limited; and (3) extraction of the original data is incomplete, and some data cannot be converted due to the lack of relevant data.

In summary, this is the first meta-analysis demonstrating the efficacy of tocilizumab treatment in severely ill COVID-19 patients.

\begin{tabular}{|c|c|c|c|c|c|c|c|c|c|}
\hline Study or Subgroup & $\begin{array}{l}\text { Tocilizu } \\
\text { Events }\end{array}$ & $\begin{array}{l}\text { mab } \\
\text { Total }\end{array}$ & \multicolumn{2}{|c|}{ Control } & Weight & \multirow{2}{*}{$\begin{array}{c}\begin{array}{c}\text { Odds Ratio } \\
\text { M-H, Fixed, 95\% Cl }\end{array} \\
0.37[0.11,1.23]\end{array}$} & \multicolumn{3}{|c|}{$\begin{array}{c}\text { Odds Ratio } \\
\text { M-H, Fixed, 95\% Cl }\end{array}$} \\
\hline Campochiaro C & 5 & 32 & 11 & 33 & $5.2 \%$ & & & $T$ & \\
\hline Capra R & 2 & 62 & 11 & 23 & $8.8 \%$ & $0.04[0.01,0.19]$ & $\longleftarrow$ & & \\
\hline Colaneri M & 5 & 21 & 19 & 91 & $3.1 \%$ & $1.18[0.38,3.64]$ & & & \\
\hline Gokhale Y & 33 & 70 & 61 & 91 & $16.0 \%$ & $0.44[0.23,0.83]$ & $\longrightarrow$ & & \\
\hline Guaraldi G & 13 & 179 & 73 & 365 & $25.4 \%$ & $0.31[0.17,0.58]$ & $\longrightarrow$ & & \\
\hline Klopfenstein T & 5 & 20 & 12 & 25 & $4.6 \%$ & $0.36[0.10,1.30]$ & & - & \\
\hline Moreno-Pérez O & 10 & 77 & 3 & 159 & $1.0 \%$ & $7.76[2.07,29.10]$ & & & \\
\hline Potere $\mathrm{N}$ & 2 & 40 & 11 & 40 & $6.0 \%$ & $0.14[0.03,0.68]$ & & & \\
\hline Rojas-Marte GR & 43 & 96 & 55 & 97 & $17.2 \%$ & $0.62[0.35,1.09]$ & & & \\
\hline Somers EC & 14 & 78 & 27 & 76 & $12.8 \%$ & $0.40[0.19,0.84]$ & - & & \\
\hline Total $(95 \% \mathrm{Cl})$ & & 675 & & 1000 & $100.0 \%$ & $0.47[0.36,0.60]$ & 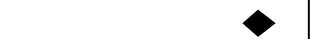 & & \\
\hline Total events & 132 & & 283 & & & & & & \\
\hline $\begin{array}{l}\text { Heterogeneity: } \mathrm{Chi}^{2}= \\
\text { Test for overall effect }\end{array}$ & $\begin{array}{l}34.76, d f \\
Z=5.76\end{array}$ & $\begin{array}{l}=9(P \\
(P<0.0\end{array}$ & $\begin{array}{l}<0.0001 \\
00001)\end{array}$ & 1); $I^{2}=$ & $74 \%$ & & $0.01 \quad 0.1 \quad 1$ & $\begin{array}{cc}10 \\
\text { Favours Control }\end{array}$ & 100 \\
\hline
\end{tabular}




\section{Abbreviations}

ACEl: Angiotension converting enzyme inhibitors; ARB: Angiotension receptors blockers; ARDS: Acute respiratory distress syndrome; COVID19: Coronavirus disease 2019; Cl: Confidence intervals; CRS: Cytokine release syndrome; $\mathrm{FiO}_{2}$ : Fraction of inspired oxygen; ICU: Intensive care unit; IL6: Interleukin-6; IMV: Invasive mechanical ventilation; NSAID: Non-steroidal anti-inflammatory drugs; NIV: Non- invasive Ventilation; OR: Odds ratio; $\mathrm{PaO}_{2}$ : Partial pressure of oxygen; $\mathrm{SaO}_{2}$ : Oxygen saturation; SARS-CoV-

2: Severe acute respiratory syndrome coronavirus 2

\section{Acknowledgements}

Not applicable.

\section{Authors' contributions}

TBP contributed the conception and design of this review; ZJ wrote the paper. TBP and CW revised and edited this manuscript. All authors reviewed the draft and approved the final manuscript for submission.

\section{Funding}

This work was supported by the Zhejiang Provinicial Natural Science Foundation of China (LY2OH010002) and the Medical and Health Research Program of Zhejiang Province (2019RC181) to TBP.

Availability of data and materials

All data generated or analyzed during this study are included in this published article.

\section{Ethics approval and consent to participate}

Not applicable.

\section{Consent for publication}

Not applicable.

\section{Competing interests}

The authors declare no conflict of interest.

Received: 29 July 2020 Accepted: 3 August 2020

Published online: 27 August 2020

\section{References}

1. Reddy SG. Population health, economics and ethics in the age of COVID-19. BMJ Glob Health. 2020;5. https://doi.org/10.1136/bmjgh-2020-003259.

2. Cully M. Immune status could determine efficacy of COVID-19 therapies. Nat Rev Drug Discov. 2020;19:431-4. https://doi.org/10.1038/d41573-020-00110-3.

3. Wiersinga WJ, Rhodes A, Cheng AC, et al. Pathophysiology, transmission, diagnosis, and treatment of coronavirus disease 2019 (COVID-19): a review. JAMA. 2020. https://doi.org/10.1001/jama.2020.12839.

4. Giamarellos-Bourboulis EJ, Netea MG, Rovina N, et al. Complex immune dysregulation in COVID-19 patients with severe respiratory failure. Cell Host Microbe. 2020;27:992-1000.e3. https://doi.org/10.1016/j.chom.2020.04.009.

5. Koutsakos M, Kedzierska K. A race to determine what drives COVID-19 severity. Nature. 2020;583:366-8. https:/doi.org/10.1038/d41586-020-01915-3.

6. Zhu LN, Yang PH, Zhao YZ, et al. Single-cell sequencing of peripheral blood mononuclear cells reveals distinct immune response landscapes of COVID19 and influenza patients. Immunity. 2020. https://doi.org/10.1016/j.immuni. 2020.07.009

\section{Publisher's Note}

Springer Nature remains neutral with regard to jurisdictional claims in published maps and institutional affiliations.

Ready to submit your research? Choose BMC and benefit from:
- fast, convenient online submission
- thorough peer review by experienced researchers in your field
- rapid publication on acceptance
- support for research data, including large and complex data types
- gold Open Access which fosters wider collaboration and increased citations
- maximum visibility for your research: over 100M website views per year
At BMC, research is always in progress.
Learn more biomedcentral.com/submissions

